\title{
Quenched Eguchi-Kawai model revisited
}

\author{
Herbert Neuberger®* \\ Department of Physics and Astronomy, Rutgers University, Piscataway, New Jersey 08854, USA
}

(Received 25 September 2020; accepted 12 October 2020; published 6 November 2020)

\begin{abstract}
The motivation and construction of the original quenched Eguchi-Kawai (QEK) model are reviewed, providing much greater detail than in the first, 1982 QEK paper. A 2008 article announced that QEK fails as a reduced model because the average over permutations of eigenvalues stays annealed. It is shown here that the original quenching logic naturally leads to a formulation with no annealed average over permutations.
\end{abstract}

DOI: $10.1103 /$ PhysRevD.102.094503

\section{INTRODUCTION}

In 1982 Eguchi and Kawai (EK) [1] made the spectacular observation that $S U(N)$ lattice gauge theory with no matter fields gave Wilson loop expectation values that were reproducible on a minimal $1^{4}$ lattice at leading order in $1 / N$. Intensive research ensued. It was found that large $N$ phase transitions caused difficulties in approaching the continuum limit. Some cures were suggested. The purpose of this paper is to make explicit the underlying logic and definitions of one of them: the quenched Eguchi-Kawai (QEK) conjecture [2]. It will be shown that the 2008 article [3] does not establish failure of the general idea of quenching. A determination of the validity or failure of quenched reduction requires future extensive numerical work.

\section{EGUCHI-KAWAI REDUCTION IS NOT JUST A LATTICE PECULIARITY}

At first sight EK reduction seemed to be an incredible lattice trick. Only much more recently has numerical work indicated that EK reduction is a property of the continuum planar four-dimensional $S U(N)$ pure gauge theory [4]. At leading order in $1 / N$ the expectation values of Wilson loops on an infinite Euclidean four-torus are the same as "folded" counterparts on any finite torus with a side size larger than about one Fermi in QCD terms.

In retrospect, this sharpens the original question that motivated [2]: how is a Coulomb-like law realized in a finite periodic Euclidean four volume which has no room for "Faraday's flux lines" to spread?

Continuum EK reduction is some times referred to as "partial reduction" because the lattices one has to use in a

\footnotetext{
*herbert.neuberger@gmail.com
}

Published by the American Physical Society under the terms of the Creative Commons Attribution 4.0 International license. Further distribution of this work must maintain attribution to the author(s) and the published article's title, journal citation, and DOI. Funded by SCOAP ${ }^{3}$. numerical simulation in order to approach the continuum limit to reasonable accuracy must be substantially larger than $1^{4}$. "Partial" is a bad epithet because it emphasizes a detail of implementation and conceals the physical content of the result.

\section{LATTICE FIX OF EK REDUCTION}

The first conjectured fix for lattice EK reduction to an $1^{4}$ lattice was the quenched EK model, QEK [2].

The main physics question was how the largeness of the $S U(N)$ group at infinite $N$ could provide a placeholder for an infinite lattice while consisting of just four unitary link matrices. A simple calculation at one loop order showed that the eigenvalue phases of the link matrices in the four directions could play the role of continuous lattice momenta in $(-\pi, \pi]^{4}$ — an "emerging" toroidal momentum space — and produce the standard Coulomb's force law on the lattice if we summed up the contributions of a large number of saddles and ignored their instabilities. As was very well understood from other semiclassical calculations, fluctuations in the "flat" directions, connecting the saddles, produced zero modes and were easily dealt with. But, the saddles in the integral were dominated by coalescing eigenvalues, so perturbation theory was unstable. As a whole, the matrix integral was benign.

On an $1^{4}$ lattice the eigenvalue sets of each link matrix are gauge invariant angles. They are unique candidates for lattice momenta.

The QEK fix consisted of a removal of the link matrices' eigenvalues from the set of annealed variables. They were quenched instead. This difference did not matter at large $N$ by a degrees-of-freedom counting argument. There were $4 N$ angles, and order $4 N^{2}$ matrix elements; the angles ought to be governed by uniform and uncorrelated distributions in each direction if some obvious symmetries remain preserved as $N \rightarrow \infty$.

For strong lattice coupling (small " $\beta$ "), where EK had been proven to work, the quenching prescription would have no effect to leading order in $\frac{1}{N}$. Quenched or annealed, 
the angle distributions would be frozen to continuous uniform densities in each direction and those would be uncorrelated. The requirement to match onto the original EK version, which was proven to hold at strong coupling, left little freedom for constructing QEK. The "loop equations," on which the EK proof relied, have trivial boundary conditions in the strong coupling limit and determine the entire strong coupling series. That series has a finite radius of convergence. The precise boundary conditions for the lattice loop equations at weak coupling remain unknown to date. They would be needed for constructing the Feynman series for Wilson loops. The loop equations themselves have only a relatively formal continuum limit. They do not offer a reliable tool for analysis in continuum directly.

\section{QUENCHING IN DETAIL}

QEK was originally presented as a conjecture, and this remains its status to date. It is uncertain whether it is valid throughout the bridge connecting short and long distance pure gauge theory physics. Even if it does, there remains doubt whether it would be practical in comparison to the safer continuum EK method which relies both on the lattice loop equations and on some numerical, nonperturbative tests.

The QEK prescription is explained below in detail and at an elementary level.

\section{A. Quenching "with calculus"}

The lattice variables we shall deal with are phase angles and unitary matrices.

The first step in constructing the quenching prescription consists of a proper change of integration variables in the EK case. For simplicity, we consider $U(N)$-restricting to $S U(N)$ later presents little difficulty. The change of variables requires a one-to-one relation between the old and new, together with a matching of integration domains. The domains in the new variables are obviously important and will be discussed later below.

The variable change is an EK $\rightarrow$ QEK map replacing each of the link variables $U_{\mu}, \mu=1,2,3,4$ by a set of angles $\theta_{\mu}^{i}, i=1, \ldots, N$ and a unitary matrix $V_{\mu}$. For four fixed $U_{\mu}$ 's, there are multiple solutions. To determine domains of integration in the new variables requires selecting one unique branch among them,

$$
\text { (1) } U_{\mu} V_{\mu}=V_{\mu} D_{\mu}\left(\theta_{\mu}\right) ; \quad \text { (2) } U_{\mu}=V_{\mu} D_{\mu}\left(\theta_{\mu}\right) V_{\mu}^{\dagger},
$$

where

$$
D_{\mu}\left(\theta_{\mu}\right)=\operatorname{diag}\left(e^{i \theta_{\mu}^{1}}, e^{i \theta_{\mu}^{2}}, \ldots e^{i \theta_{\mu}^{N}}\right) .
$$

The original integration measure is

$$
\prod_{\mu=1,2,3,4} d U_{\mu} \quad \text { where } d U_{\mu} \text { is Haar. }
$$

After the change of variables the integration measure is locally given by

$$
\prod_{\mu=1,2,3,4}\left[d V_{\mu} \prod_{i=1, \ldots . N} d \theta_{\mu}^{i} \prod_{i<j} \sin ^{2}\left(\frac{\theta_{\mu}^{i}-\theta_{\mu}^{j}}{2}\right)\right],
$$

up to a constant factor determined by a normalization convention. The Jacobian does not depend on $V$, only on the angles. This is crucial for the quenching proposal because the integration over the angles factorizes. In EK the instability resides in the angle dynamics. The QEK model addresses the instability directly.

The $U_{\mu}$ 's are uniquely determined by the $\theta$ 's and $V$ 's: the $\theta_{\mu}$ exponents are the eigenvalues of $U_{\mu}$ and the columns of $V_{\mu}$ are the corresponding eigenvectors. The multiple solutions of equality (2) in Eq. (4.1) with given $U$ are all generated from each other by group transformations. This makes the search for unique branches straightforward. Two types of maps between solutions enter and together they exhaust their multiplicity:

First, one may multiply equality (1) in Eq. (4.1) by a $\mu$-dependent diagonal matrix from the right. This multiplies each column of $V_{\mu}$ by a phase: eigenvectors are rays. The transformations are a product of commuting $U(1)^{N}$ 's, one for each direction. Second, one may multiply equality (1) in Eq. (4.1) from the right by a permutation matrix $P \in S(N) \subset U(N) . P$ is a unitary matrix having a single entry equal to 1 in each row and each column. When acting on a column from the left, it permutes its row entries by $P . P^{\dagger}$ acts from the right on a row and permutes its column entries by $P^{-1}$. Inserting $1=P P^{\dagger}$ on the right-hand side of Eq. (1) in (4.1) between the $\mathrm{V}$ and the $\theta$-diagonal matrix replaces $V$ by $V P$ on both sides. The effect of $P^{\dagger} \ldots . . P$ on the diagonal $\theta$ matrix is to permute its columns by $P$ and its rows by $P^{\dagger}$. The result is that the $\theta_{\mu}^{i}$ get permuted by $P$ along the diagonal. Thus, the elements $P \in S(N)$ act simultaneously on the columns of $V$ and on their associated eigenvalues, preserving the eigenvalue-eigenvector association.

Suppose one is given a $U$ matrix. By a probability argument, it has distinct eigenvalues. A procedure to identify a unique decomposition in terms of $V$ and $\operatorname{diag}(\theta)$ matrices is defined as follows: one first finds all the roots of the characteristic polynomial of $U$. Then, for each eigenvalue, one finds a corresponding unit norm column eigenvector. Arranging the columns left to right, one gets a unitary matrix $V$. This $V$ has its columns further reordered into a canonical form. This form will be defined below. The positions of the angles along diagonals of the $D$ matrices are fixed by the order of the columns of the matrix $V$.

We learned that the domain of integration in $V$ and $\theta$ variables is the $S(N)$ right coset space [5],

$$
\left[U(N) / U(1)^{N} \times U(1)^{N}\right] / S(N) .
$$

Factoring by $S(N)$ defines an equivalence relation $\sim$ in the space of the new variables which partitions $U(N) / U(1)^{N} \times$ $U(1)^{N}$ into equivalence classes. The domain of integration 
consists of one specific representative of each class. The intention is to make a choice that has a chance to protect the perturbatively emerging momentum space from the instability: We choose in each class a representative element of the matrix component, $V$, close to the unit matrix. This element consists of an ordered set of column eigenvectors of the corresponding $U$-matrix. It can also be viewed as a choice of an ordered orthonormal basis whose elements correspond to a set of $N$ angles determining the eigenvalues of the corresponding $U$ matrix. The order of the eigenvalues is determined by the order of the columns of $V$. The solution for $V$ and $\theta$ of equality (2) in (4.1) has become unique.

The prescription suggested here is that $V$ have the entry of maximal absolute value along the portion of each row from the diagonal to the right on the diagonal. Equal absolute values are ignored for probability reasons. That entry is made real positive by a phase choice for the corresponding column. A given $V$ can be brought into this configuration sequentially, starting from the top row and bringing the desired entry to the $(1,1)$ location by permuting through $N$ columns, and then continuing to the next row and permuting through the $N-1$ remaining columns to bring the desired element of the row to the $(2,2)$ location and so on. Real positivity fixes the phase of each column. It mods out the factor $U(1)^{N}$ in the "numerator" of (4.5). For the lack of a better term, let us call this ordering "diagonally right row entry dominant". This clumsy name avoids confusion with the standard term "diagonal dominance." With probability one, this choice is unambiguous.

In the EK case, at any finite $N$, which specific choice of representative was made does not matter because the integral over ordered angles and bases is done on one common integrand and the $U(1)^{N}$ and $S(N)$ are symmetries of the integrand. In the QEK case however, the integral over the angles occurs at a later stage, with a modified integrand and the choice does matter because $S(N)$ acts simultaneously on $\theta$ 's and $V$ 's. At finite $N$ quenched expectation values depend on which representative of each equivalence class was chosen. This is a crucial feature of quenching.

The correct ranges of integration in QEK are over a set of four $V$ 's, all in "canonical" order. A criterion for choosing the canonical order is that the $V$ representative be "perturbative." There are no restrictions on the angles. Different orders of the same set are included as separate contribution. There exist other prescriptions that are equally valid. To be sure, for large lattice $\beta$ couplings the reduction validity of QEK remains a conjecture.

Permutations of eigenvectors must be eliminated in order to perform a correct variable change in EK with no overcounting at any coupling. That is just applied multivariate calculus.

To motivate the ordering prescription for the $V$ matrices, consider the simple case of $U(2) / S(2)$. The two $U(1)^{2}$ terms in the "numerator" of Eq. (4.5) are ignored. Up to an irrelevant overall phase, any $V \in U(2)$ can be written as

$$
V=\left(\begin{array}{cc}
w & z^{*} \\
-z & w^{*}
\end{array}\right),
$$

where $|w|^{2}+|z|^{2}=1 . S(2)=\left\{1_{2}, \sigma_{1}\right\}$ using Pauli's notation. Let $V^{\prime}$ be given by the same expression with $w, z$ replaced by $w^{\prime}, z^{\prime}$. The equation $V=V^{\prime} \sigma_{1}$ defines an equivalent pair $V^{\prime} \sim V$. In components, $z^{\prime}=-w^{*}$ and $w^{\prime}=-z^{*}$. For $V$ to be "diagonally right row entry dominant" we need $|w|>|z|$; then $V^{\prime}$ is not "diagonally right row entry dominant" because $\left|w^{\prime}\right|<\left|z^{\prime}\right|$. The split of $U(2)$ into "halves" is explicit. The half containing the identity makes up the QEK integration domain for $V$. As usual, ambiguous cases are ignored for probability reasons.

$U(N)$ can be restricted to $S U(N)$ by adding a factor of $\delta_{2 \pi}\left(\sum_{i} \theta_{\mu}^{i}\right)$ in the $\theta$ integral for each direction. $\delta_{2 \pi}$ is the $2 \pi$-periodic $\delta$ function. Mentally, one can imagine the $V$ 's to be also fixed by an overall phase, restricting to $S U(N)$; this phase does not enter observables dependent only on the $U$ 's.

Once the change of variables in the EK integrand is correctly implemented one can replace each $U_{\mu}$ by equality (2) in Eq. (4.1) in the EK model and nothing has changed. The integral for the partition function can be done successively, first integrating over all the columns of the $V_{\mu}$ in the pair $\left[\theta_{\mu}^{i}, i\right.$ th column of $V_{\mu}$ ] with local Haar measure at fixed, ordered, angles at each $\mu$. Next, one integrates over all of the possible ordered angle sets for each $\mu$.

Quenching replaces the EK by QEK. In QEK the integral for the partition function is replaced by an integral with the same measure and action, but at fixed angles in all directions. The QEK partition function is a function of these angles, $Z(\theta)$. For a Wilson loop observable one uses $Z(\theta)$ as normalization, now in the denominator, obtaining averages of the observable at a fixed ordered $\theta$ set. Next, these annealed $V$-averages are integrated over the angles with weight given by the Jacobian in Eq. (4.4). The $\theta$ variables are treated as a set of random couplings, akin to the $J$-couplings of a spin-glass model.

The traditional choice for angle ordering is descending along the diagonal with values in the segment $(-\pi, \pi]$. Such an ordering makes the distribution equal to the derivative of a smooth approximation to the angle dependence on the index in each direction separately. ${ }^{1}$ But, this is not permitted in QEK. The order of the angles cannot be restricted in any way. One has a diagonalizing ordered basis, and one can assign to each eigenvector an eigenvalue on the unit circle, distributed just according to the Jacobian factor. The Jacobian measure is invariant under direction dependent permutations. They are not induced by annealed

\footnotetext{
${ }^{1}$ See Eq. 10 in [6].
} 
generation of permutations among the columns of $V$ because two distinct "diagonally right row entry dominant" $V$-matrices cannot be related by a nontrivial permutation.

There is no invariance under the hypercubic group at fixed $\theta$. It could happen that new large $N$ transitions occur, to phases where the hypercubic symmetry is spontaneously broken. Such phases indeed do occur in the EK model [4]. If they persist to the quenched case, QEK fails. It also could happen that it is practically impossible to attain high enough values of $N$ because prohibitively large samples of the eigenvalue sets are needed for a reasonable accurate estimate of the final angle integral.

The ordering of $V$ determines that of $V^{\dagger} . V^{\dagger}$ is not "diagonally right row entry dominant." The action controlling the $V$ average depends only on the six combinations $V_{\mu \nu}=V_{\nu \mu}^{\dagger} \equiv V_{\mu}^{\dagger} V_{\nu}$ for $\mu>\nu$ [2]. These are overlap matrices of ordered eigenvector-sets corresponding to the angles in the $\mu, \nu$ directions. The common canonical ordering of the $V_{\mu}$ 's induces some preference for the $V_{\mu \nu}$ 's to be closer to identity with no direct feedback on the angles-unlike in the EK situation. The hope is that angles are now free to take on the role of an "emergent" lattice momentum space.

\section{V. “GAUGE INVARIANCE” IN QEK}

On an $1^{4}$ lattice, gauge theory has a symmetry under simultaneous conjugation by the same matrix of all link matrices. These matrices can be thought of as Polyakov loops. Evidently, on a one site lattice there can be no geometrically open contours.

This symmetry acts on the $V$-matrices from the left and therefore commutes with the action on $V$ by the $S(N)$ we had to mod out by. A permutation gauge transformation will permute the rows of each $V$. After its action each $V$ needs to be reordered back to canonical order. The end result is that gauge transformations which happen to be permutations do not change anything. We might as well forget about them altogether.

\section{A. Conclusion of section}

This paper contains the full description of the original, with no shortcuts allowed, QEK model. There are many ways and points of view in which QEK can fail. To the limited extent I understand it, the Bringolz-Sharpe paper [3] has not analyzed a precise enough version of the originally intended QEK model. If I am right, the problem of inprinciple validity of QEK remains open. Numerical tests might discover a new candidate problem with QEK in the future which could invalidate the quenching approach in principle.

\section{FINAL COMMENTS}

In this paper algorithmic issues in the QEK case have not been addressed. Clearly, the $U(2)$ example was presented with traditional $S U(2)$ Monte Carlo updates in mind. An HMC (hybrid Monte Carlo) version might be also worth looking into.

From the extensive and ultimately successful work on the twisted EK model [7], TEK, it is known that for TEK to work, the large $N$ limit needs to be approached with care and one needs to go to truly large values of $N$. By the law of "conservation of difficulty" QEK may also need further nontrivial refinements. The problem of annealed permutations Bringoltz and Sharpe [3] found, at least at the theoretical level, seems harmless to me because the basic rules of calculus would tell you to eliminate permutations in the quenching approach and how to do it.

\section{ACKNOWLEDGMENTS}

I am grateful to Barak Bringolz for providing me, many years ago, with a succinct formulation of the main point of the Bringoltz and Sharpe paper [3], namely, that Eq. (10) in [2] showed that there was an issue with permutations that could jeopardize the validity of QEK. I thank Rajamani Narayanan for valuable discussions and comments. Support by the sabbatical program of the Schwartz/ Reisman Institute for Theoretical Physics at the Weizmann Institute of Science is gratefully acknowledged.
[1] T. Eguchi and H. Kawai, Reduction of Dynamical Degrees of Freedom in the Large- $N$ Gauge Theory, Phys. Rev. Lett. 48, 1063 (1982).

[2] G. Bhanot, U. M. Heller, and H. Neuberger, The quenched Eguchi-Kawai model, Phys. Lett. 113B, 47 (1982).

[3] B. Bringoltz and S. R. Sharpe, Breakdown of large- $N$ quenched reduction in $S U(N)$ lattice gauge theories, Phys. Rev. D 78, 034507 (2008).

[4] J. Kiskis, R. Narayanan, and H. Neuberger, Does the crossover from perturbative to nonperturbative physics in QCD become a phase transition at infinite $N$ ?, Phys. Lett. B 574, 65 (2003).

[5] B. Eynard, T. Kimura, and S. Ribault, Random matrices, arXiv: $1510.04430 \mathrm{v} 2$.

[6] D. J. Gross and E. Witten, Possible third-order phase transition in the large- $N$ lattice gauge theory, Phys. Rev. D 21, 446 (1980).

[7] For a recent review see M. G. Perez, Prospects for large N gauge theories on the lattice, Proc. Sci., LATTICE2019 (2020) 276 [arXiv:2001.10859], and references therein. 